\title{
Fluorescent and bioluminescent calcium indicators with tuneable colors and affinities
}

Nicole Mertes ${ }^{1}$, Marvin Busch ${ }^{1}$, Magnus-Carsten Huppertz ${ }^{1}$, Christina Nicole Hacker ${ }^{1}$, Clara-Marie Gürth$^{2}$, Stefanie Kühn¹, Julien Hiblot ${ }^{1}$, Birgit Koch ${ }^{1}$, Kai Johnsson ${ }^{1,3^{*}}$

${ }^{1}$ Department of Chemical Biology, Max Planck Institute for Medical Research, Jahnstrasse 29, 69120 Heidelberg, Germany

${ }^{2}$ Department of Optical Nanoscopy, Max Planck Institute for Medical Research, Jahnstrasse 29, 69120 Heidelberg, Germany

${ }^{3}$ Institute of Chemical Sciences and Engineering, École Polytechnique Fédérale de Lausanne (EPFL), 1015 Lausanne, Switzerland

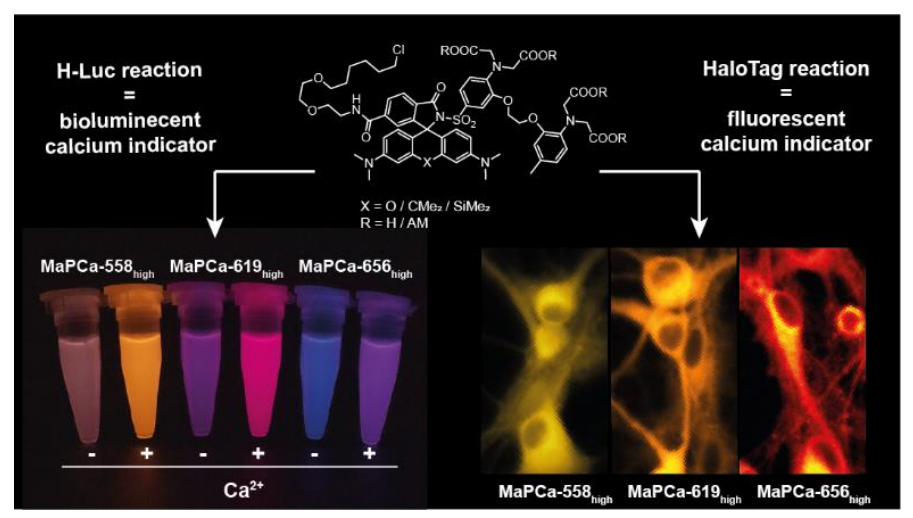

Graphical abstract

Email: johnsson@mr.mpg.de

KEYWORDS: Fluorescence, Bioluminescence, Calcium Indicator

\section{Abstract}

We introduce a family of bright, rhodamine-based calcium indicators with tuneable affinities and colors. The indicators can be specifically localized to different cellular compartments and are compatible with both fluorescence and bioluminescence readouts through conjugation to HaloTag fusion proteins. Importantly, their increase in fluorescence upon localization enables no-wash livecell imaging, which greatly facilitates their use in biological assays. Applications as fluorescent indicators in rat hippocampal neurons include the detection of single action potentials and of calcium fluxes in the endoplasmic reticulum (ER). Applications as bioluminescent indicators include the recording of the pharmacological modulation of nuclear calcium in high-throughput- 
compatible assays. The versatility and remarkable ease of use of these indicators make them powerful tools for bioimaging and bioassays.

\section{Introduction}

The second messenger calcium is involved in a plethora of signaling pathways and biochemical processes. ${ }^{1}$ The elucidation of its function in cellular processes has become possible largely through the development of calcium indicators. ${ }^{2-4}$ While early development focused on synthetic calcium indicators, genetically encoded calcium indicators (GECIs) have now become the gold standard. The main reason for this is that GECls can be genetically targeted to specific cellular populations and subcellular localizations, whereas the cellular uptake of synthetic calcium indicators lacks selectivity and is often inefficient. However, GECls possess lower brightness, slower response kinetics and a limited color range (especially in the far-red in comparison to synthetic indicators). 5,6 These limitations are of particular concern when highly localized areas, such as micro- and even nanodomains are investigated, and more demanding microscopy techniques are used..$^{7-9} \mathrm{~A}$ possibility to combine the brightness, response kinetics and spectral range of synthetic fluorescent indicators with the targetability of GECls is the use of self-labeling protein tags such as SNAP-tag and HaloTag. ${ }^{10,11}$ Self-labeling proteins form a covalent bond to a specific substrate and through this enable precise localization of synthetic molecules to proteins of interest (POI). This approach has been used to create a number of localizable synthetic calcium indicators, e.g. BG3-Indo-1, ${ }^{12}$ BOCA-1-BG ${ }^{13}$ or RhoCa-Halo ${ }^{14}$ and the far-red indicator $\mathrm{JF}_{646^{-}}$ BAPTA. ${ }^{5,15}$ However, these probes have limited cell permeability and solubility, and furthermore require washing steps to remove unreacted probes, greatly limiting their applicability. ${ }^{13,14}$ The use of bright synthetic fluorophores for calcium sensing was enabled developing chemogenetic sensors in which the protein-based calcium-sensing domain Calmodulin (CaM) interacts with an environmentally sensitive dye (e.g. rHCaMP or HaloCaMP). ${ }^{16,17}$ However, based on the same calcium sensing domain as most GECls, they suffer from relatively slow response kinetics. ${ }^{16}$ Furthermore, there is currently no localizable synthetic far-red calcium indicator with a suitable calcium affinity for calcium-rich areas like the endoplasmic reticulum (ER) or calcium microdomains. ${ }^{18,19}$ Here we present MaPCa dyes, a family of highly permeable calcium indicators with different colors and calcium affinities that can be coupled to HaloTag. As the reaction with HaloTag shifts the fluorescent scaffold of the indicator from a non-fluorescent into a fluorescent configuration, these probes can be used without any washing steps to remove unbound probe. 

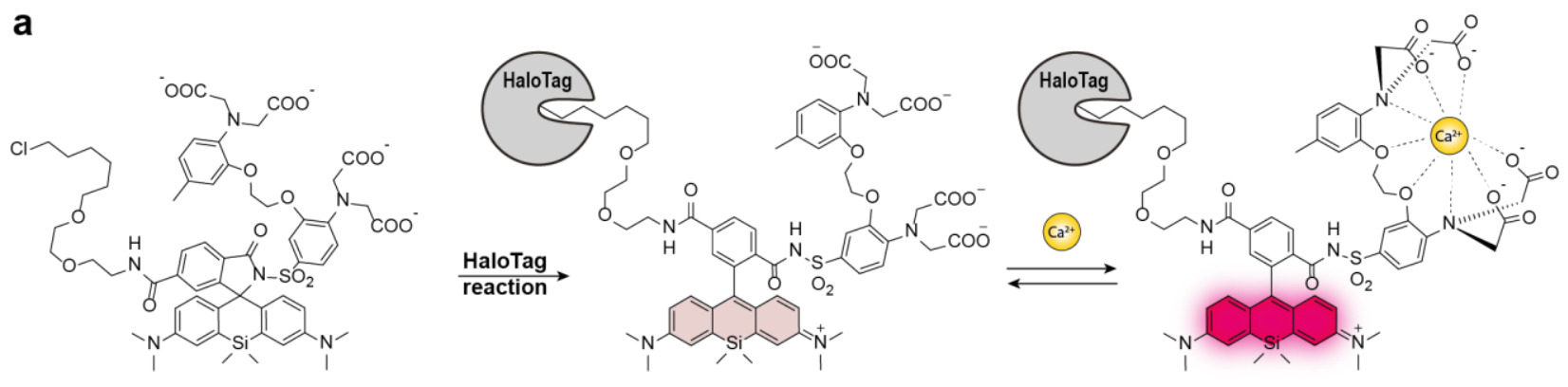

b

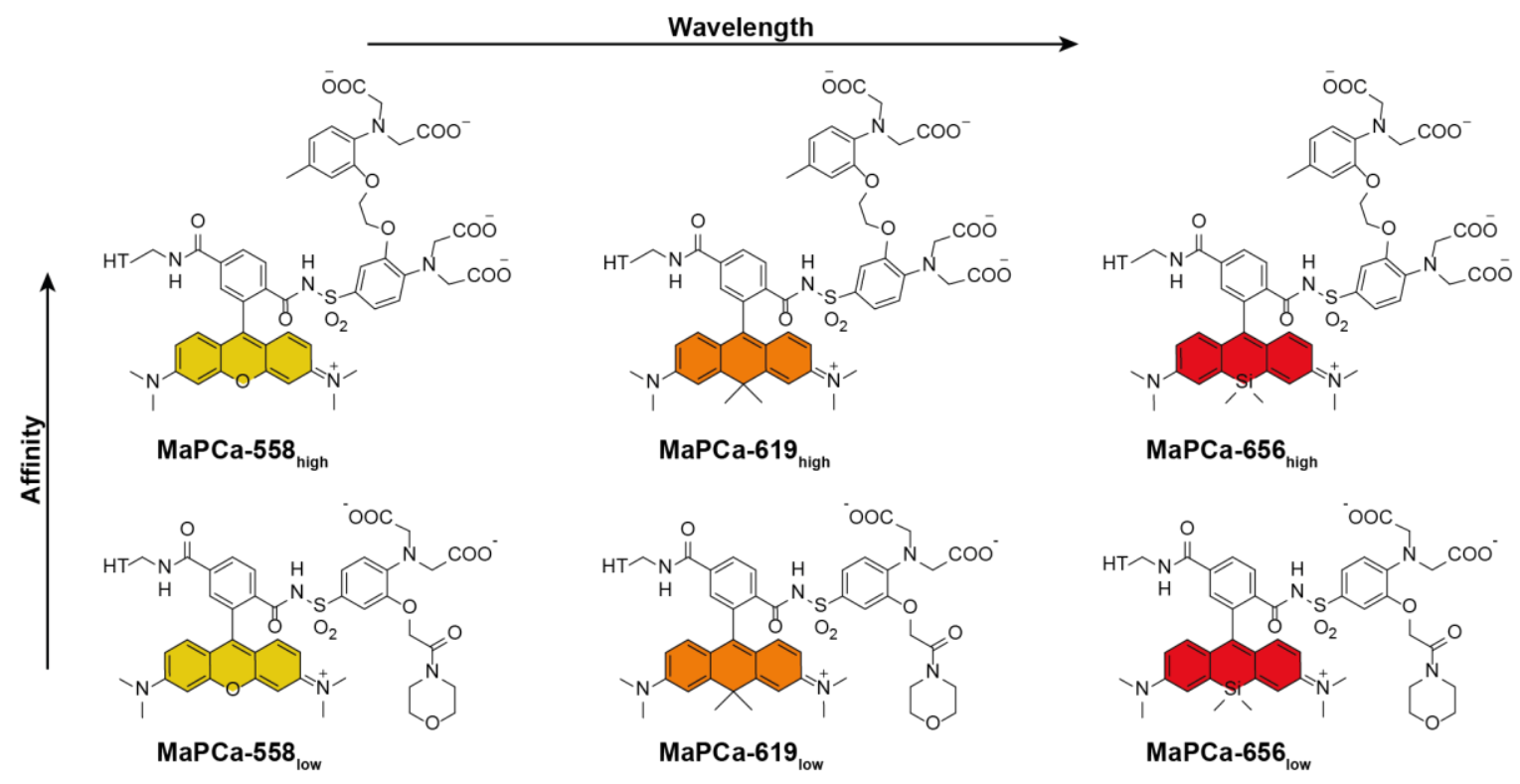

Fig. 1: Schematic representation of the MaPCa dyes. a) Representation of the double-turn-on mechanism of MaPCa dyes. Example for MaPCa-656high. If not bound to the HaloTag, MaPCa dyes are in their colorless, spirocyclic form. Upon binding to HaloTag, they open to their zwitterionic form and hence become potentially fluorescent, but PETquenched by the $\mathrm{Ca}^{2+}$-binding moiety. Only upon calcium binding full fluorescence is achieved. b) Overview of synthesized MaPCa dyes. HT= HaloTag-bound linker.

\section{Results and discussion}

\section{Design principle, synthesis and in vitro characterization of MaPCa dyes}

The design of our calcium indicators is based on the recently introduced MaP dyes, in which the lactone-forming carboxylic acid of a rhodamine is replaced with an amide attached to an electron withdrawing group (e.g. sulfonamides). ${ }^{20,21}$ This results in dyes that preferentially exist as a nonfluorescent spirolactam in solution, but shift to an open, fluorescent state upon binding to HaloTag, enabling no-wash imaging with low background. We envisioned designing fluorogenic calcium indicators by attaching a calcium chelator such as BAPTA (1,2-bis(o-aminophenoxy)ethane$N, N, N$ ', $N^{\prime}$-tetraacetic acid) through a benzene sulfonamide to the ortho-carboxylate of rhodamines and a chloroalkane (CA) through a carboxylate at the 6-position of the benzyl-ring (Fig. 1). BAPTA would be thereby positioned in close proximity to the rhodamine core, which is an important factor 
for effective PET-quenching of the rhodamine by the free chelator. ${ }^{15,22}$ Attachment of the CA via the 6-position of the benzyl-ring would enable HaloTag to shift the equilibrium from spirocyclization to the fluorescent, open form, thereby resulting in fluorogenicity. Furthermore, attachment of the CA via the 6-position would ensure a high labeling speed of the resulting HaloTag substrate. ${ }^{23}$

We set out to synthesize a set of such indicators based on the high-affinity calcium chelator BAPTA and the low-affinity chelator MOBHA (2-(2'-morpholino-2'-oxoethoxy)- $N, N$ bis(hydroxycarbonylmethyl) aniline) ${ }^{24}$ in combination with commercially available rhodamine-CA substrates TMR-CA, CPY-CA and SiR-CA, covering the spectrum from 550 to $650 \mathrm{~nm}$ (Fig. 2). In a first step, a sulfonamine was attached to the previously described BAPTA-ethylester ${ }^{25}$ (01) or

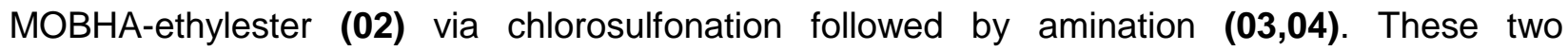
intermediates were then coupled to the commercially available rhodamine-CAs TMR-CA, CPY$\mathrm{CA}$ and SiR-CA using activation by chlorosulfonic acid. The indicators were obtained as free acids after saponification with $\mathrm{KOH}$ (Fig. 2a). For in cellulo experiments, acetoxymethyl (AM) esters of the indicators were synthesized by prior transesterification of the chelator $(05,06)$ and subsequent coupling to the fluorophore. The AM-esters serve to mask the carboxylic acids to ensure cellpermeability, but are cleaved inside the cell by endogenous esterases. ${ }^{26}$ We named these indicators MaPCa dyes (for Max-Planck-Calcium sensor), with a postfix expressing the absorption maxima in $\mathrm{nm}(\mathrm{TMR}=558$; $\mathrm{CPY}=619 ; \mathrm{SiR}=656)$ and the subscripts 'high' or 'low' for indicating the calcium affinity range. The AM-esters of the dyes are marked with an additional AM, in contrast to the saponified probes. It should be noted that this short and convergent synthetic scheme should enable the conversion of most rhodamine-CAs into calcium sensors in a single step. 

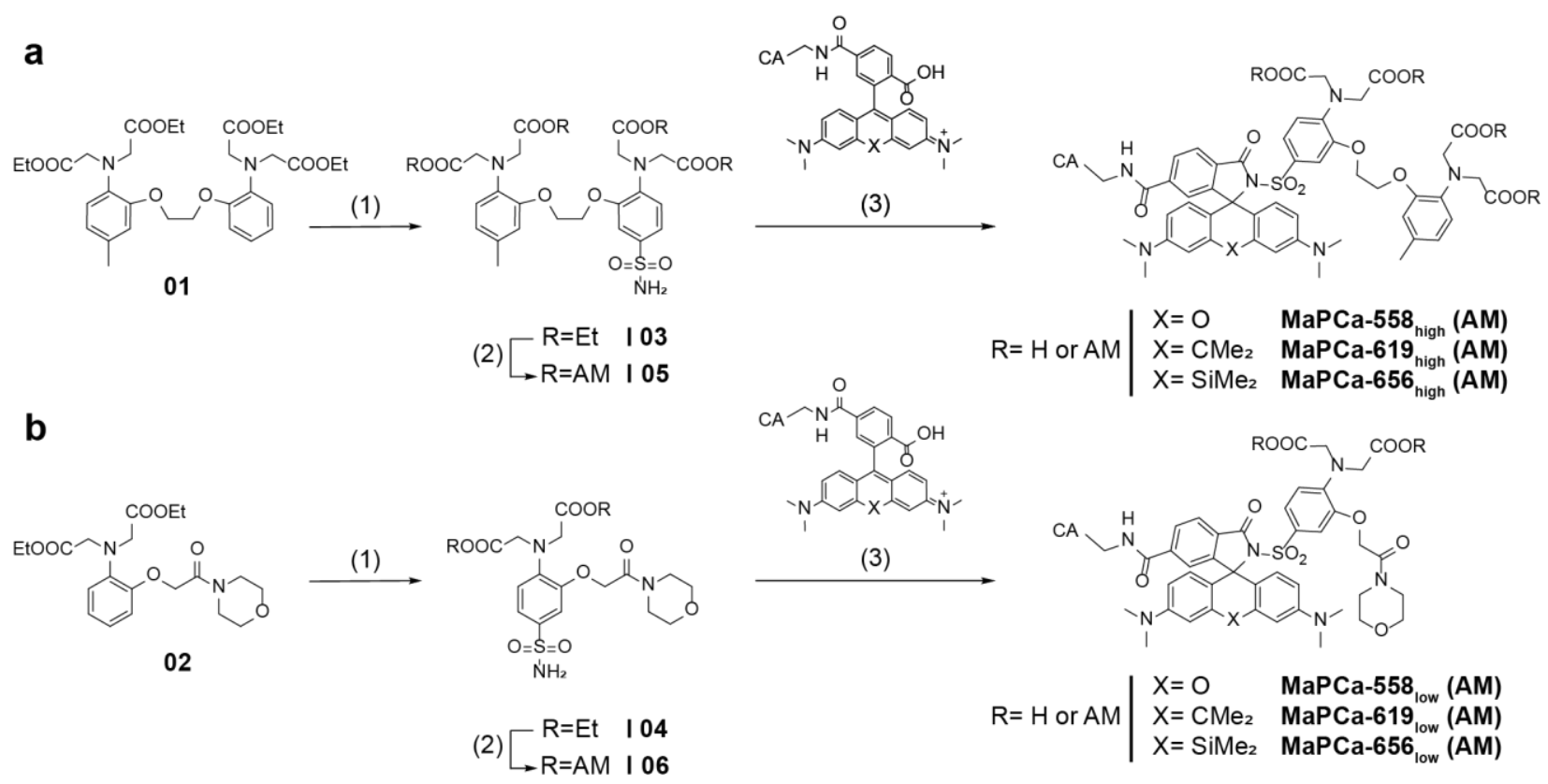

b

Fig. 2: Synthetic pathway for the preparation of MaPCa dyes. The AM esters of the dyes are marked with an additional $\mathrm{AM}$, in contrast to the saponified probe. (a) Synthetic route for $\mathrm{MaPCa}_{\text {high }}$ (1) i) $\mathrm{HSO}_{3} \mathrm{Cl}, \mathrm{SOCl}_{2}, \mathrm{DCM}^{\circ} 0^{\circ} \mathrm{C}-\mathrm{rt}, 24 \mathrm{~h}$ ii) aq. $\mathrm{NH}_{3}$, EtOAc, rt, 75\%; (2) this step was only performed for the AM-probes for the cellular experiments: i) DMAP, di-tertbutyl-dicarbonate, DCM, $35^{\circ} \mathrm{C}, 24 \mathrm{~h}$ ii) $\mathrm{KOH}, \mathrm{MeOH} / \mathrm{THF}, \mathrm{rt}, 2 \mathrm{~h}$ iii) DIPEA, bromomethyl acetate, MeCN, rt, $48 \mathrm{~h}$ iv) TFA, DCM, rt, 2 h, 61\%; (3) i) fluorophore preactivation with $\mathrm{SOCl}_{2}$, pyridine, $\mathrm{DCM}, \mathrm{rt}-60^{\circ} \mathrm{C}, 0.5 \mathrm{~h}$ ii) DIPEA, DMAP, $60^{\circ} \mathrm{C}, 1 \mathrm{~h}, 26-56 \%$; the ethylester was subsequently saponified: $\mathrm{KOH}$, MeOH/THF, rt, $8 \mathrm{~h}, 42-66 \%$. (b) Synthetic route for $\mathrm{MaPCa}_{\text {low }}$ (1) i) $\mathrm{HSO}_{3} \mathrm{Cl}, \mathrm{SOCl}_{2}, \mathrm{DCM}, 0^{\circ} \mathrm{C}$-rt, $24 \mathrm{~h}$ ii) aq. $\mathrm{NH}_{3}$, EtOAc, rt, 44\%; (2) this step was only performed for the AM-probes for the cellular experiments: i) DMAP, di-tert-butyl-dicarbonate, $\mathrm{DCM} / \mathrm{MeCN}, 35^{\circ} \mathrm{C}, 43 \mathrm{~h}$ ii) $\mathrm{KOH}$, $\mathrm{MeOH} / \mathrm{THF}, \mathrm{rt}, 5.5 \mathrm{~h}$ iii) DIPEA, bromomethyl acetate, MeCN, rt, $21 \mathrm{~h}$ iv) TFA, DCM, TIPS, rt, 0.5 h, 36\%; (3) i) fluorophore preactivation with $\mathrm{SOCl}_{2}$, pyridine, DCM, rt $-60^{\circ} \mathrm{C}, 0.5 \mathrm{~h}$ ii) DIPEA, DMAP, $60^{\circ} \mathrm{C}, 3.5 \mathrm{~h}, 13-32 \%$; the ethylester was subsequently saponified: $\mathrm{KOH}, \mathrm{MeOH} / \mathrm{THF}, \mathrm{rt}, 5 \mathrm{~h}, 22-58 \%$.

The MaPCa dyes calcium responsiveness was characterized in vitro in presence and absence of HaloTag measuring their fluorescence intensity at different free calcium concentrations (Fig. 3a, 3b, Supporting Fig. 3,4). As desired, all three high-affinity indicators showed a fluorogenic turn-on upon binding to HaloTag. However, while MaPCa-558 high $_{\text {was }}$ only slightly fluorogenic (1.3-fold), MaPCa-619 high $_{\text {and MaPCa-656 }}$ high showed a significant 7-fold and even 120-fold increase upon binding to HaloTag, respectively, in the calcium-bound state. The higher fluorogenicity of MaPCa$656_{\text {high }}$ can be rationalized considering the higher propensity of SiR derivatives to exist in the nonfluorescent spirocyclic form than the corresponding rhodamine and carborhodamine derivatives. ${ }^{27}$ The dyes possess a high brightness in the calcium-bound state (quantum yield $>40 \%$; extinction coefficient $>80^{\prime} 000 \mathrm{M}^{-1} \mathrm{~cm}^{-1}$ ) and display calcium-affinities in a suitable range for cytosolic measurements $\left(\mathrm{K}_{\mathrm{D}}\left(\mathrm{Ca}^{2+}\right)\right.$ : 410-580 $\left.\mathrm{nM}\right)$ with turn-ons of around 6-fold upon calcium binding (Table 1, Supporting Table 1). The low-affinity indicators show similar fluorogenicities as the BAPTA-variants: the TMR-variant (MaPCa-558 low) shows low fluorogenicity (1.4-fold) upon HaloTag binding, while MaPCa-619 low (28-fold) and MaPCa-656 low (208-fold) are highly fluorogenic. The calcium affinities of these dyes are in the range of $220-460 \mu \mathrm{M}$ and they show a 
7 to 11-fold turn-on upon calcium binding (Table 1, Supporting Table 1). The extinction coefficient of MaPCa-656 $6_{\text {low }}$ is significantly lower than those of the other MaPCa-indicators, suggesting that it does not fully convert to the open state. Nevertheless, its brightness of $\sim 15 \mathrm{mM}^{-1} \mathrm{~cm}^{-1}$ is in the same order of magnitude than genetically encoded red-shifted indicators (brightness FRGECO1c: $\left.9.3 \mathrm{mM}^{-1} \mathrm{~cm}^{-1}\right) .28$

Table 1: Photophysical properties of MaPCa dyes. HT= HaloTag.

\begin{tabular}{|c|c|c|c|c|c|c|c|}
\hline & \multicolumn{2}{|c|}{$\mathrm{F} / \mathrm{F}_{0}$} & \multirow{3}{*}{$\begin{array}{c}\lambda \mathrm{Ex} / \lambda \mathrm{Em} \\
{[\mathrm{nm}]}\end{array}$} & \multirow{3}{*}{$\begin{array}{c}\mathrm{K}_{\mathrm{D}}\left(\mathrm{Ca}^{2+}\right) \\
{[\mu \mathrm{M}]^{*}} \\
\text { HT-bound }\end{array}$} & \multirow{2}{*}{$\begin{array}{c}\varepsilon \\
{\left[\mathrm{mM}^{-1} \mathrm{~cm}^{-1}\right]}\end{array}$} & \multirow[t]{2}{*}{$\Phi$} & \multirow{3}{*}{$\begin{array}{l}\text { Brightness } \\
{\left[\mathrm{mM}^{-1} \mathrm{~cm}^{-1}\right]}\end{array}$} \\
\hline & $\mathrm{Ca}^{2+}$-bound & HT-bound & & & & & \\
\hline & $\begin{array}{l}\text { Upon HT7- } \\
\text { binding }\end{array}$ & $\begin{array}{c}\text { Upon } \mathrm{Ca}^{2+}- \\
\text { binding }\end{array}$ & & & \multicolumn{2}{|c|}{$\mathrm{Ca}^{2+}$-bound / HT-bound } & \\
\hline MaPCa-558high & 1.3 & 6 & $558 / 580$ & 0.41 & 81 & $49 \%$ & 40 \\
\hline MaPCa-619 high & 7 & 6 & $619 / 632$ & 0.57 & 91 & $60 \%$ & 55 \\
\hline MaPCa-656 high & 120 & 6 & $656 / 670$ & 0.58 & 81 & $41 \%$ & 33 \\
\hline MaPCa-558low & 1.4 & 7 & $560 / 580$ & 224 & 68 & $38 \%$ & 26 \\
\hline MaPCa-619low & 28 & 8 & $618 / 633$ & 322 & 93 & $48 \%$ & 45 \\
\hline MaPCa-656low & 208 & 11 & $655 / 670$ & 457 & 34 & $44 \%$ & 15 \\
\hline
\end{tabular}

We hypothesized that the increase of fluorescence intensity of the MaPCa dyes upon calcium binding should be mainly due to decreased PET quenching. However, MaPCa-656 ${ }_{\text {high }}$ and MaPCa656 low show a $20-30 \%$ increase in absorbance upon calcium binding (Fig. 3c). This can be rationalized considering that both indicators, when bound to the HaloTag in the absence of calcium, are not fully in the open state. Calcium binding then weakens the electron-donating effect of the aniline moiety, pushing the equilibrium further to the open, fluorescent state (Supporting Fig. 5).

\section{In-cellulo characterization of the MaPCa dyes}

For first cellular calcium imaging experiments, AM-esters of the MaPCanigh indicators were applied to co-cultures of 293 cells stably expressing a nuclear localized HaloTag and 293 cells without HaloTag. Imaging the cells without any washing steps after $2 \mathrm{~h}$ incubation already revealed efficient HaloTag labeling (Fig. 3d), demonstrating that these molecules are cell permeable. The comparison of the cytosolic background fluorescence intensity in non-expressing cells vs. the

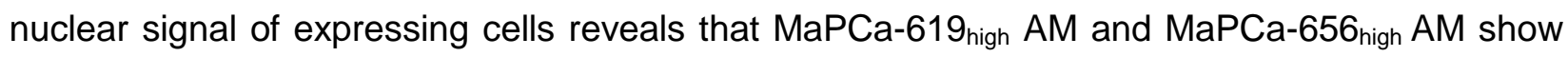
excellent signal to background ratios $\left(F_{\text {nud }} / F_{\text {cyt }}=6\right.$ and 9 , respectively) (Fig. 3d). This can be 
rationalized by the high fluorogenicity of these two substrates. In contrast, the low fluorogenicity

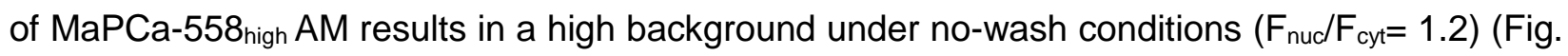
3d). Furthermore, all MaPCa ${ }_{\text {high }} \mathrm{AM}$ indicators translated the calcium concentration rise induced by ATP treatment by a mean fluorescence intensity increase $\left(\Delta F / F_{0}\right)$ ranging between 0.5 and 2 . (Fig. 3e, Supporting Fig. 6).

a

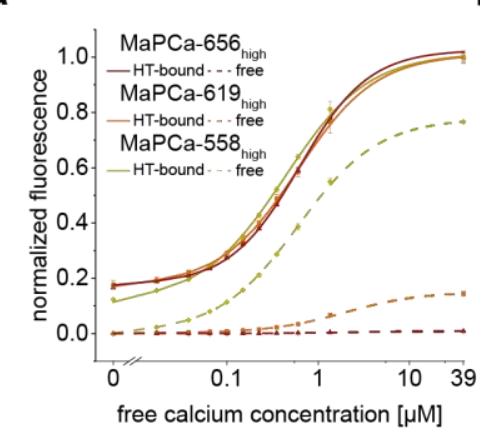

d

b

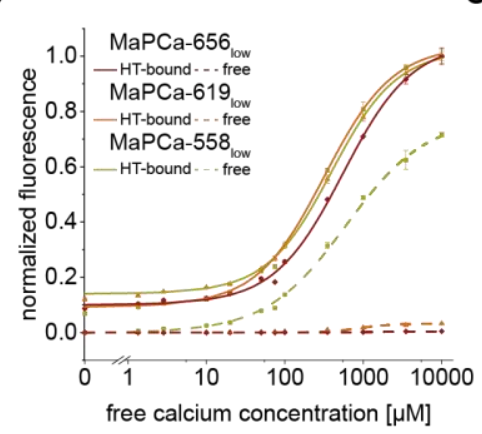

e
C
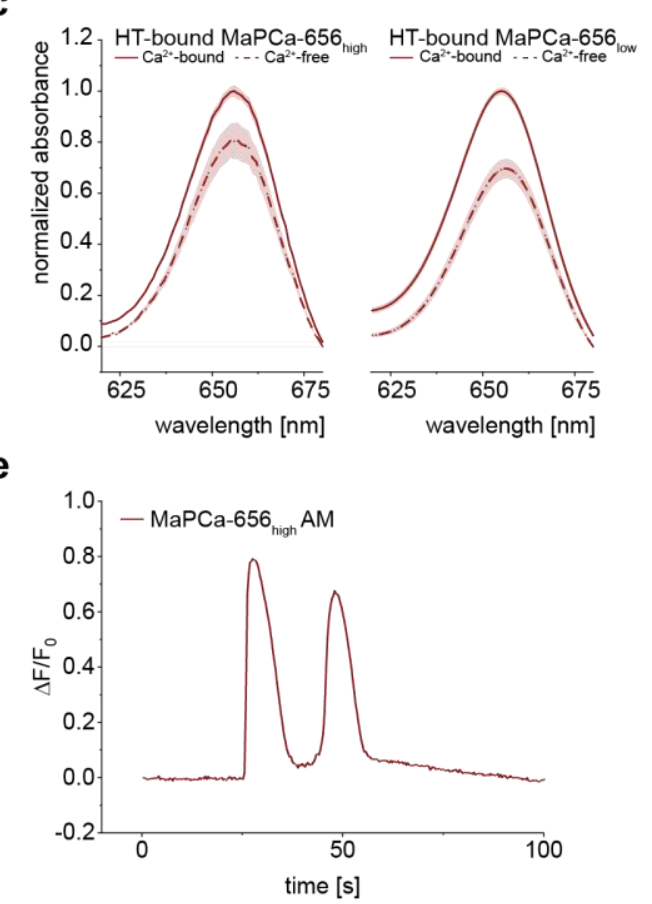

Fig. 3: Characterization of MaPCa dyes. (a,b) Calcium titration of (a) MaPCanigh and (b) MaPCalow. (c) Absorbance spectra of HT-bound MaPCa-656 indicators show calcium dependent absorbance increase. (d) Fluorescence microscopy images of a co-culture of HaloTag-NLS-expressing and non-expressing 293 cells. Cells were incubated with $1 \mu \mathrm{M}$ MaPCa-558high AM (left), MaPCa-619 high AM (middle) or MaPCa-656high AM (right) for $2 \mathrm{~h}$ and imaged under nowash conditions. Turn-on numbers represent average of $n=200$ cells. Scale bars: $20 \mu \mathrm{m}$. (e) Exemplary fluorescence trace of 293 stably expressing HaloTag-SNAP-tag fusion protein in the nucleus, incubated with MaPCa-656high AM and perfused with $100 \mu \mathrm{M}$ ATP. HT= HaloTag.

\section{MaPCa dyes report on calcium signaling in neurons}

In a next step, the performance of the MaPCa indicator series was evaluated in rat primary hippocampal neurons. For experiments with primary neuronal cultures, the possibility to perform the labeling without any washing steps is important, as such steps are known to disturb viability of primary cell cultures. ${ }^{29}$ rAAV transduced rat primary hippocampal neurons expressing HaloTagmEGFP strictly in the cytoplasm were labeled with either MaPCa-619 high AM or MaPCa-656 $_{\text {high }}$ AM and imaged under no-wash conditions. Both dyes led to efficient and homogeneous HaloTag labeling without the occurrence of a significant background signal or unspecific staining. While usable, MaPCa-558 high $_{\text {AM required a washing step to reach results similar to MaPCa-619 }}$ high AM

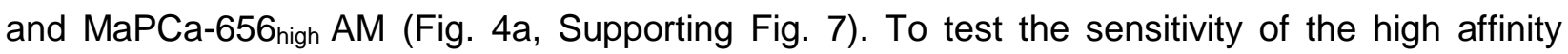


MaPCa indicators, labeled neurons were stimulated with a distinct number of action potentials (APs) using electric field stimulation. ${ }^{30}$ All dyes allowed the detection of a single AP with $\Delta \mathrm{F} / \mathrm{F}_{0}$ values ranging between $3 \%$ (MaPCa-558 high $_{\text {AM) }}$ ) and $6 \%$ (MaPCa-656 high $\mathrm{AM}$ ), while $\Delta \mathrm{F} / \mathrm{F}_{0}$ of

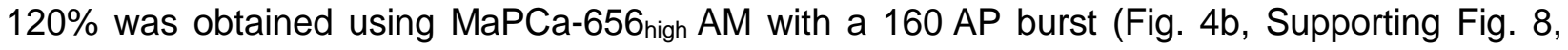
Supporting Video 1).

The lower calcium affinity of the MaPCalow series allows to report calcium fluctuations in compartments with high basal calcium concentrations such as the ER $\left(\mathrm{Ca}^{2+}\right.$ conc.: $\left.500 \mu \mathrm{M}\right){ }^{19}$ Therefore, the MaPCa dyes were target to the ER through rat hippocampal neuron transduction localizing a HaloTag-SNAP-tag fusion in the ER. Co-staining of SNAP-tag confirmed efficient and specific labeling of HaloTag with MaPCalow dyes under no-wash conditions, with the exception of MaPCa-558 $8_{\text {low }}$ AM that required a washing step to reduce background (Supporting Fig. 9). The ER is a calcium store which, upon stimulation, can release calcium into the cytosol. Here, the RyR2 channel plays a crucial role as a calcium-induced calcium-release channel. ${ }^{31}$ As the red-shifted wavelengths of the MaPCa dyes do not spectrally overlap with the GFP channel, we multiplexed the MaPCa signal from the ER with a cytosolic GCaMP6f, i.e. to simultaneously image calcium efflux from the ER and cytosolic influx upon stimulation. Specifically, rat hippocampal neurons were double transduced using rAAVs expressing both constructs individually and then labeled with the MaPCa $\mathrm{M}_{\text {low }} \mathrm{AM}$ indicators. Upon addition of caffeine, a RyR2 stimulant, ${ }^{31,32}$ we could simultaneously record a signal decrease in the ER due to calcium efflux (MaPCa $\left.a_{\text {low }} A M\right)$ and a concomitant signal increase in the cytosol due to calcium influx (GCaMP6f) (Fig. 4 c,d, Supporting Fig. 10, Supporting Video 2). This demonstrates how MaPCa AM dyes allow, in combination with established GCaMP sensors, to visualize the complex interplay between calcium pools in different cellular compartments in a time-resolved manner. 
a

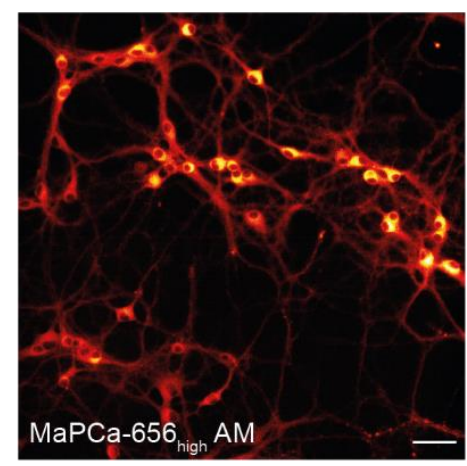

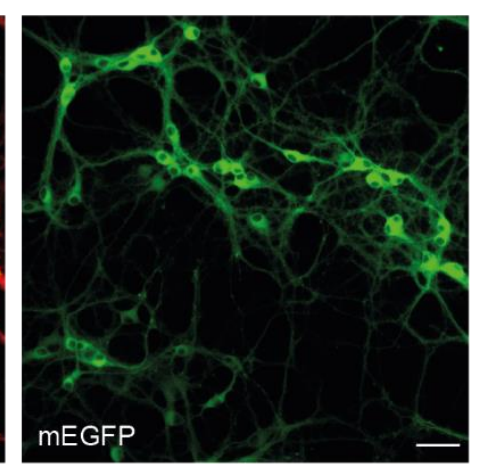

C
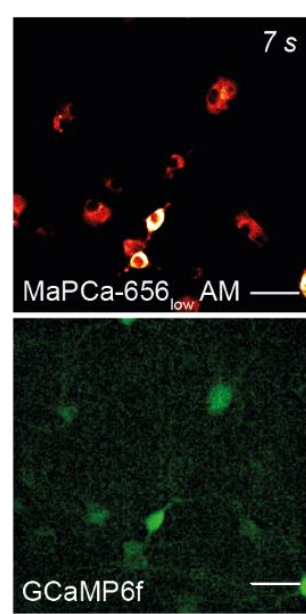
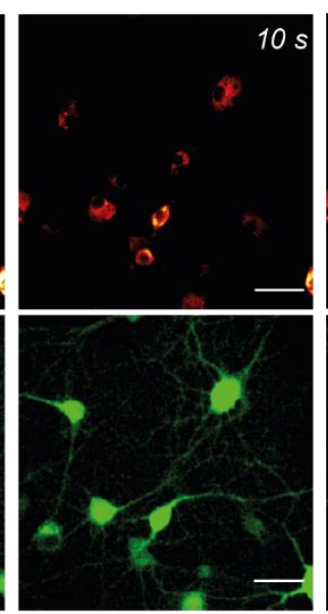

b

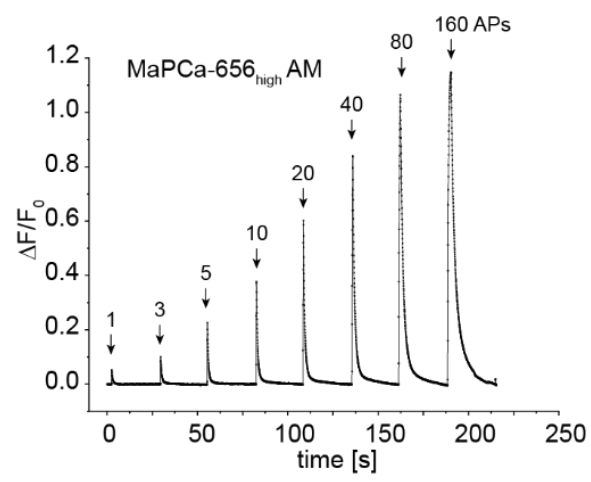

d

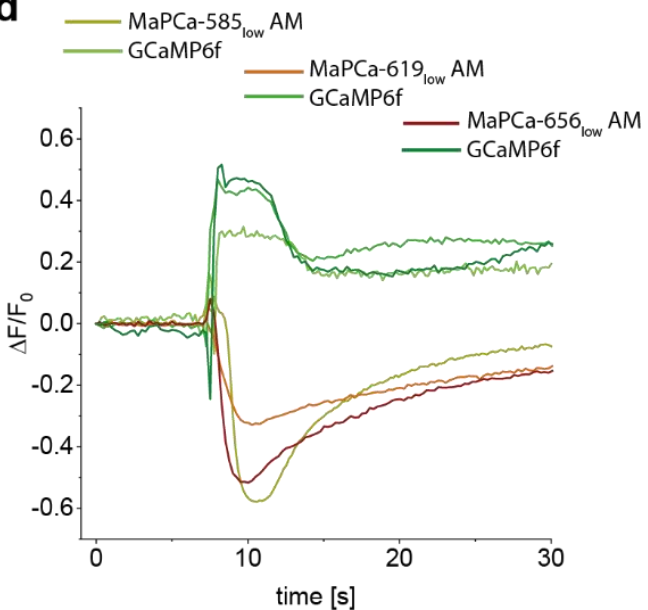

Fig. 4: MaPCa dyes can report on calcium flux in primary rat hippocampal neurons. (a) Fluorescence microscopy images of primary rat hippocampal neurons expressing NES-HaloTag-eGFP incubated with $1 \mu \mathrm{M}$ MaPCa-656high AM and imaged under no-wash conditions; MaPCa-656 high-channel (left) and eGFP-channel (right). Scale bar $50 \mu \mathrm{m}$. (b) Baseline-corrected average trace of stimulated neurons expressing HaloTag and incubated with $1 \mu \mathrm{M}$ MaPCa-656high AM under no-wash conditions ( $\mathrm{n} \geq 50$ cells). APs: 1,2,5,10,20,40,80,160. (c) Fluorescence microscopy images of rat hippocampal neurons expressing ER-localized HaloTag and cytosolic GCaMP6f. Cells were incubated with $1 \mu \mathrm{M}$ MaPCa-656 low AM for $2 \mathrm{~h}$ and imaged under no-wash conditions. After $\sim 7 \mathrm{~s}$ caffeine (final conc.: $20 \mathrm{mM}$ ) was added. (d) Fluorescence time trace of a representative cell in (c) and of identically treated cells with the indicators MaPCa558 low AM and MaPCa-619 low AM (single representative cell) imaged simultaneously with GCaMP6f. Scale bar $50 \mu \mathrm{m}$.

\section{Bioluminescence as a readout}

The MaPCa dyes could potentially also be used for the labeling of H-Luc, a chimera between HaloTag and the furimazine-dependent luciferase NanoLuc. ${ }^{33}$ Labeling of $\mathrm{H}$-Luc with rhodamine dyes can result in efficient BRET from NanoLuc to the bound rhodamine, such that emission at both $450 \mathrm{~nm}$ and at the emission wavelength of the bound rhodamine can be observed. We hypothesized that labeling H-Luc with MaPCa dyes would lead to the development of bioluminescent calcium indicators with tunable emission wavelengths with up to far-red light emission (Fig. 5a). Existing bioluminescent calcium indicators, such as Orange CAMBI, ${ }^{34}$ GLICO, ${ }^{35}$ LUCI-GECO $1,{ }^{36} \mathrm{CeNL}^{37}$ or CalfluxVTN ${ }^{38}$ rely exclusively on fluorescent proteins that possess emission maxima restricted below $600 \mathrm{~nm}$. We therefore labeled $\mathrm{H}$-Luc with the MaPCa dyes and recorded the emitted light upon addition of furimazine in the absence and presence of 
calcium. As is already apparent by eye (Fig. 5b), the color of the emitted light dramatically depends on both, the presence of calcium as well as the nature of the MaPCa dye attached to H-Luc (Fig.

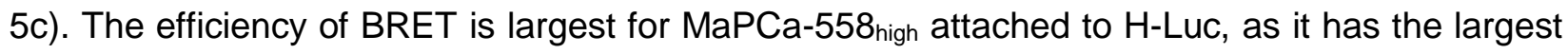
spectral overlap with the BRET donor. As the intensity of the emission of the MaPCa dye depends on the concentration of calcium, measuring the ratio of the intensity of emitted light at $450 \mathrm{~nm}$ versus the intensity of the light emitted at the emission maximum of the rhodamine dye can thus be used to record changes in calcium concentrations (Fig. 5b, c, Supporting Fig. 11). The maximal change in ratio ranged from 6.5 for $\mathrm{H}$-Luc labeled with MaPCa-656 high $_{\text {to }} 4.2$ for $\mathrm{H}$-Luc labeled with MaPCa-619 high. The H-Luc-MaPCa ratio changes are comparable to those of previously described ratiometric, bioluminescent calcium sensors and to the best of our knowledge, H-Luc labeled with

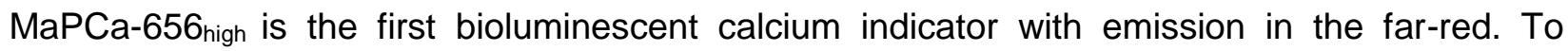
demonstrate how these ratiometric bioluminescent calcium sensors can be exploited for cellular applications, Flp-In 293 cells with a nuclear H-Luc expression were labeled with the MaPCa $\mathrm{M}_{\text {high }}$ AM dye series. The cells were then exposed to a solution of ATP and thapsigargin and the emission ratio of the emitted light was recorded. A significant change in luminescence emission ratio for all three MaPCa dyes was observed upon drug treatment, the value being the highest for

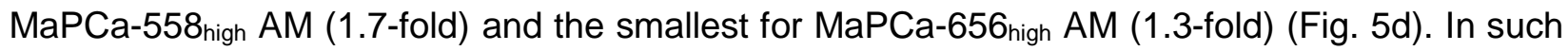
given experimental conditions, each channel luminescence intensity was integrated in less than $500 \mathrm{~ms}$, allowing changes in calcium concentrations to be followed with good temporal resolution. The z-factor is a measure for the statistical effect size used to judge the suitability of an assay for high-throughput screening (HTS) approaches. 293-cells expressing H-Luc labeled with MaPCa558 high AM presented a z-value of 0.58 upon ATP/thapsigargin treatment, highlighting the suitability of such bioassay for HTS (z-factors $\geq 0.5$ indicate excellent suitability). ${ }^{39}$ Finally, lowaffinity bioluminescent calcium indicators could be generated by labeling $\mathrm{H}$-Luc with the MaPCa $\mathrm{a}_{\text {low }}$ indicators, demonstrating the modularity of the approach (Supporting Fig. 12). 

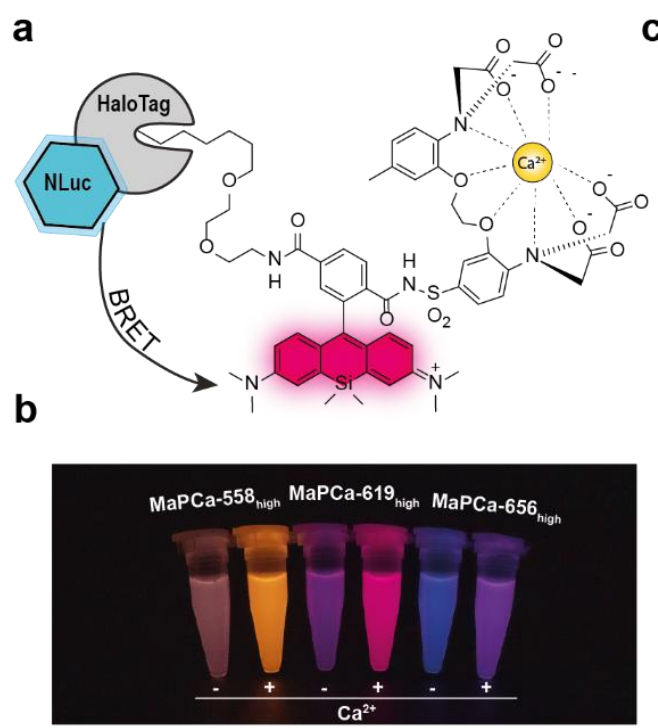

C

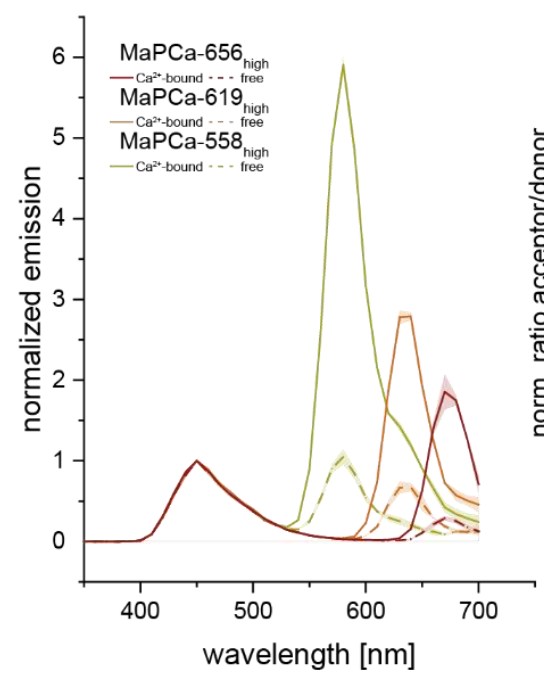

d

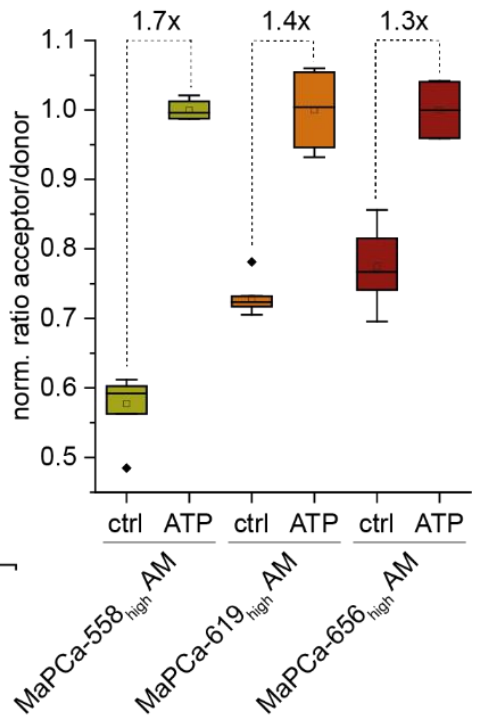

Fig. 5: Characterization of MaPCanigh based bioluminescent indicators. (a) Bioluminescent H-Luc transfers energy (BRET) to bound MaPCa dyes. (b) Picture of Eppendorf tubes filled with H-Luc labeled MaPCanigh dyes in the absence or presence of calcium. (c) Normalized in vitro emission spectra of $\mathrm{H}$-Luc labeled MaPCanigh dyes, with-and without calcium. (d) Normalized acceptor-donor ratio of 293 cells expressing H-Luc in the nucleus and labeled with $1 \mu \mathrm{M}$ MaPCanigh AM-dyes. Shown is the ratio of control wells and wells treated of $100 \mu \mathrm{M}$ ATP and $5 \mu \mathrm{M}$ thapsigargin $(\mathrm{N} \geq 4$ wells per condition).

\section{Conclusion}

We have introduced a new design principle for the development of localizable and fluorogenic calcium indicators. Using this strategy, we have developed several indicators with different colors, up to the far-red, and with different calcium affinities. What distinguishes these indicators from previous work is the good permeability of the probes and the possibility to use them without additional washing steps to remove unbound indicator. This greatly facilitates their use in most biological applications. Furthermore, they are accessible through a short and modular synthetic pathway. We demonstrated applications of the indicators in rat hippocampal neurons, where the high-affinity indicator MaPCanigh could detect single APs under no-wash conditions. The lowaffinity indicator $\mathrm{MaPCa}_{\text {।ow }}$ was successfully localized in the $\mathrm{ER}$, where it could detect calcium efflux isochronal to increase in cytosolic calcium detected by GCaMP6f. We furthermore developed the first far-red bioluminescent calcium indicator by coupling MaPCa with H-Luc, a bioluminescent HaloTag. The use of H-Luc-MaPCa in cells also demonstrated the possibility to use such bioassays in HTS approaches. These examples underscore the versatility of these calcium indicators and their ease of use.

Finally, the established design principles of these calcium indicators should be transferable to analytes other than calcium. 


\section{Methods}

Detailed procedures for the synthesis of all compounds, their characterization and imaging experiments are given in the Supplementary Information.

General. For all microscopy images, a DMi8 widefield microscope (Leica) equipped with a HC PL APO 20x/0.8 (dry) was used. Excitation/Emission settings: TMR-based dyes: $\lambda$ ex $=515 / 30 \mathrm{~nm}$, detection $\lambda$ det $=609 / 54 \mathrm{~nm}$; CPY $/ \mathrm{SiR}$ based dyes: $\lambda e x=635 / 18 \mathrm{~nm}$, detection $\lambda$ det $=700 / 75 \mathrm{~nm}$. Imaging data was processed using Fiji. ${ }^{40}$ ROls were drawn manually and average fluorescence intensities extracted and plotted using Origin software. HaloTag in this work corresponds to the variant HaloTag7.

Signal/background measurements FIp-In 293 cells. Flp-In 293 cells stably expressing HaloTag-SNAP-NLS (see Supporting Information) were co-cultured with non-expressing cells on a 10-well-plate (Greiner bio-one; cellview cell culture slide; glass bottom). After $2 \mathrm{~h}$ incubation with $1 \mu \mathrm{M}$ MaPCanigh AM and 0.04\% Pluronic-F127, cells were imaged without any washing steps. For analysis, $n \geq 200$ cells from at least 4 different wells were selected. Raw intensities over $\mathrm{ROI}$ of identical size were averaged $(\mathrm{Al})$ for expressing $\left(\mathrm{Al} \mathrm{I}_{\mathrm{E}}\right)$ and non-expressing $\left(\mathrm{A} \mathrm{I}_{\mathrm{NE}}\right)$ cells and signal/background were calculated by measuring the ratio $\mathrm{AlE} / \mathrm{AlNE}$.

ATP perfusion experiments. Flp-In 293 stably expressing HaloTag-SNAP-NLS (see Supporting Information) were seeded in Ibidi $\mu$-Slides (VI 0.4, Poly-L-Lysine coated, part No: 80604) $24 \mathrm{~h}$ before start of the experiment. SNAP-tag was co-stained by incubating cells with TMR-CP or SiR-BG at $1 \mu \mathrm{M}$ for $30 \mathrm{~min}$ to $1 \mathrm{~h}$ at $37^{\circ} \mathrm{C}$. After washing twice with medium, cells were stained with calcium dyes $(1 \mu \mathrm{M}+0.04 \%$ Pluronic-F127, $2 \mathrm{~h})$ through HaloTag labeling. The chamber was placed on the microscope and attached to a gravity-flow perfusion system. Cells were perfused with HBSS and, upon trigger, with HBSS containing 100 MM ATP. Image acquisition was performed every 350 ms.

Live-cell labeling and imaging in primary hippocampal neurons. Primary hippocampal neurons were prepared from postnatal P0-P2 Wistar rats as previously described ${ }^{41}$ (see also supplementary information) and cultured in 24-well glass-bottom plates for 14-18 days. The procedure was conducted in accordance with the Animal Welfare Law of the Federal Republic of Germany (Tierschutzgesetz der Bundesrepublik Deutschland, TierSchG) and the regulation for animals used in experiments (1 August 2013, Tierschutzversuchsverordnung). To euthanize rodents for the subsequent preparation of any tissue, all the regulations given in Section 4 of the TierSchG were followed. As the euthanization of animals is not an experiment on animals according to Section 7 paragraph 2 sentence 3 of the TierSchG, no specific authorization or notification is required. On day $7-8$, neurons were transduced with $0.5 \mu \mathrm{L}$ of the corresponding rAAVs for hSyn1 driven expression of NES-HaloTag-mEGFP; CalR-HaloTag-Snap-tag-KDEL (ER-localizing) or GCaMP6f (Addgene 100837- AAV1). On day 14-18 the neurons were incubated for at least $2 \mathrm{~h}$ with $1 \mu \mathrm{M}$ of the corresponding MaPCa dyes containing $0.04 \%$ Pluronic-F127. While the medium of MaPCa-558 was subsequently exchanged once, the other dyes were imaged under no-wash conditions. In case a co-stain was utilized, this was added before the MaPCa-staining at $1 \mu \mathrm{M}$ for $1 \mathrm{~h}$ followed by a single medium exchange afterwards. For stimulation of the ER-localized MaPCa dyes, a caffeine-solution (final concentration: $20 \mathrm{mM}$ ) was administered manually during recording (Frame rate: $250 \mathrm{~ms}$ ). For electric field stimulation, synaptic blockers NBQX (10 $\mu \mathrm{M}$, Santa Cruz) and APV (25 $\mu \mathrm{M}$, Sigma Aldrich) were added in order to suppress natural spiking activity. Then, a custom-built ${ }^{30}$ electrode was inserted into the wells and APs (1,2,5,10,20,40,80,160: with 25 s pauses) were evoked with following settings: Pulse width: 1 ms; Amperage 
$100 \mathrm{~mA}$; Frequency $80 \mathrm{~Hz}$. Data represents the average of $\mathrm{N} \geq 50$ manually selected $\mathrm{ROI}$ of different neurons from at least three different wells. Frame rate: $50 \mathrm{~ms}$.

Live-cell measurements with bioluminescent readout. The Flp-In System (ThermoFisher Scientific) was used to generate stable 293 cells expressing $\mathrm{H}$-Luc in the nucleus (see Supporting Information). The cells stably expressing $\mathrm{H}$ Luc-NLS were plated on a black flat glass bottom 96-well plates (Eppendorf; tissue culture treated). They were incubated with $1 \mu \mathrm{M}$ MaPCanigh AM dyes and $0.04 \%$ Pluronic-F127 for at least $2 \mathrm{~h}$ in $100 \mu \mathrm{L}$ at $37^{\circ} \mathrm{C} .50 \mu \mathrm{L}$ of a mix substrate/ extracellular inhibitor were added in each well to measure only the luminescence coming from intact cells [NanoBRET TM Nano-Glo® Substrate/ Extracellular NanoLuc $\circledast$ Inhibitor solution (final dilution substrate: 1'000x; final dilution inhibitor: 3'000x)]. Half the wells were treated with an additional $10 \mu \mathrm{L}$ of mixture ATP/thapsigargin (final concentrations ATP: $100 \mu \mathrm{M}$; thapsigargin: $5 \mu \mathrm{M}$ ). Measurements were performed stepwise (max three wells at a time, max two minutes delay between pipetting and imaging) in order to ensure identical conditions. Spectra from whole sample wells were measured on a plate reader (Spark20M, Tecan). Data represents averaged results from $\mathrm{N}=4$ experimental replicates with standard deviations. The average ratios between BRET acceptor and BRET donor of ATP/thapsigargin-treated wells were then compared to the non-treated wells.

The $z$-factor was calculated with the formula

$$
z-\text { factor }=1-\frac{3\left(\sigma_{p}+\sigma_{n}\right)}{\left|\mu_{p}+\mu_{n}\right|}
$$

With $\sigma=$ standard deviation; $\mu=$ mean; $\mathrm{p}=$ positive control and $\mathrm{n}=$ negative control.

General statistics. All measurements were measured at least in triplicates and presented as mean with s.d. if not stated otherwise. Cellular imaging experiments were performed at least twice on different days.

\section{Acknowledgements}

N.M and M.-C.H. are grateful for a Boehringer Ingelheim Fonds PhD Fellowship. This work was furthermore supported by the Max Planck Society and the Heidelberg Biosciences International Graduate School (HBIGS). N.M. was further supported by SFB grant 1129. The authors furthermore want to thank Bettina Réssy and Dominik Schmidt for the synthesis of starting materials, Andrea Bergner for plasmid/protein productions and purifications and Annette Herold for the rAAV production. Furthermore, the authors want to thank Elisa D'Este, Jasmine Hubrich, Angel Rafael Cereceda Delgado and Victor Macarrón Palacios for the support in neuronal cell culture.

\section{Contributions}

N.M. and K.J. planned the experiments and co-wrote the paper with the help of all authors. N.M. synthesized and characterized the MaPCa $a_{\text {igh }}$ dyes and performed all imaging experiments. M.B. synthesized and characterized MaPCalow. M.-C.H. and C.-M.G. assisted with neuronal cell culture 
bioRxiv preprint doi: https://doi.org/10.1101/2021.11.13.468356; this version posted November 14,2021 . The copyright holder for this preprint (which was not certified by peer review) is the author/funder, who has granted bioRxiv a license to display the preprint in perpetuity. It is made available under aCC-BY 4.0 International license.

experiments. C.H. helped to develop the synthetic pathway. S.K. and B.K generated cell lines. J.H. contributed to planning and execution of the bioluminescence experiments.

\section{Competing interests}

$\mathrm{KJ}$ is an inventor on a patent on the MaP dyes filed by the Max Planck Society.

\section{Additional Information}

Correspondences and requests for material should be addressed to K.J. 


\section{References}

1

2

3

4

5

6

7

8

9

10

Bootman, M. D. Calcium signaling. Cold Spring Harb Perspect Biol 4, a011171-a011171, (2012).

Clapham, D. E. Calcium signaling. Cell 131, 1047-1058, (2007).

Paredes, R. M., Etzler, J. C., Watts, L. T., Zheng, W. \& Lechleiter, J. D. Chemical calcium indicators. Methods 46, 143-151, (2008).

McCombs, J. E. \& Palmer, A. E. Measuring calcium dynamics in living cells with genetically encodable calcium indicators. Methods 46, 152-159, (2008).

Deo, C. \& Lavis, L. D. Synthetic and genetically encoded fluorescent neural activity indicators. Curr Opin Neurobiol 50, 101-108, (2018).

Oheim, M. et al. New red-fluorescent calcium indicators for optogenetics, photoactivation and multi-color imaging. Biochimica et Biophysica Acta (BBA) - Molecular Cell Research 1843, 22842306, (2014).

Lavis, L. D. Chemistry Is Dead. Long Live Chemistry! Biochemistry 56, 5165-5170, (2017).

Banaz, N., Mäkelä, J. \& Uphoff, S. Choosing the right label for single-molecule tracking in live bacteria: side-by-side comparison of photoactivatable fluorescent protein and Halo tag dyes. Journal of physics D: Applied physics 52, 064002, (2018).

Lock, J. T., Parker, I. \& Smith, I. F. A comparison of fluorescent $\mathrm{Ca}(2)(+)$ indicators for imaging local $\mathrm{Ca}(2)(+)$ signals in cultured cells. Cell Calcium 58, 638-648, (2015).

Los, G. V. et al. HaloTag: A Novel Protein Labeling Technology for Cell Imaging and Protein Analysis. ACS Chemical Biology 3, 373-382, (2008).

Keppler, A. et al. A general method for the covalent labeling of fusion proteins with small molecules in vivo. Nat Biotechnol 21, 86-89, (2003).

Bannwarth, M. et al. Indo-1 derivatives for local calcium sensing. ACS chemical biology 4, 179-190, (2009).

Kamiya, M. \& Johnsson, K. Localizable and Highly Sensitive Calcium Indicator Based on a BODIPY Fluorophore. Analytical Chemistry 82, 6472-6479, (2010).

Best, M. et al. Protein-specific localization of a rhodamine-based calcium-sensor in living cells. Org Biomol Chem 14, 5606-5611, (2016).

Deo, C., Sheu, S.-H., Seo, J., Clapham, D. E. \& Lavis, L. D. Isomeric Tuning Yields Bright and Targetable Red Ca2+ Indicators. Journal of the American Chemical Society 141, 13734-13738, (2019).

Deo, C. et al. The HaloTag as a general scaffold for far-red tunable chemigenetic indicators. Nature Chemical Biology 17, 718-723, (2021).

Wang, L., Hiblot, J., Popp, C., Xue, L. \& Johnsson, K. Environmentally Sensitive Color-Shifting Fluorophores for Bioimaging. Angewandte Chemie International Edition 59, 21880-21884, (2020). Samtleben, S. et al. Direct imaging of ER calcium with targeted-esterase induced dye loading (TED). Journal of visualized experiments : JoVE, e50317-e50317, (2013).

Henderson, M. J. et al. A Low Affinity GCaMP3 Variant (GCaMPer) for Imaging the Endoplasmic Reticulum Calcium Store. PLOS ONE 10, e0139273, (2015).

Wang, L. et al. A general strategy to develop cell permeable and fluorogenic probes for multicolour nanoscopy. Nature Chemistry 12, 165-172, (2020).

Lardon, N. et al. Systematic Tuning of Rhodamine Spirocyclization for Super-resolution Microscopy. Journal of the American Chemical Society 143, 14592-14600, (2021).

Turro, N., Scaiano, J. \& Ramamurthy, V. Modern Molecular Photochemistry of Organic Molecules; University Science Book: Sausalito, CA, 2010.

Wilhelm, J. et al. Kinetic and Structural Characterization of the Self-Labeling Protein Tags HaloTag7, SNAP-tag, and CLIP-tag. Biochemistry 60, 2560-2575, (2021). 
Tour, O. et al. Calcium Green FIAsH as a genetically targeted small-molecule calcium indicator. Nature Chemical Biology 3, 423-431, (2007). improved fluorescence properties. Journal of Biological Chemistry 260, 3440-3450, (1985).

Tsien, R. Y. A non-disruptive technique for loading calcium buffers and indicators into cells. Nature 290, 527-528, (1981).

27 Lukinavicius, G. et al. A near-infrared fluorophore for live-cell super-resolution microscopy of cellular proteins. Nat Chem 5, 132-139, (2013).

Dalangin, R. et al. Far-red fluorescent genetically encoded calcium ion indicators. bioRxiv, (2020).

Wardill, T. J. et al. A Neuron-Based Screening Platform for Optimizing Genetically-Encoded Calcium Indicators. PLOS ONE 8, e77728, (2013).

31 Beck, A., Nieden, R. Z., Schneider, H.-P. \& Deitmer, J. W. Calcium release from intracellular stores in rodent astrocytes and neurons in situ. Cell Calcium 35, 47-58, (2004). of Neurons. Physiological Reviews 85, 201-279, (2005).

33 Hiblot, J. et al. Luciferases with Tunable Emission Wavelengths. Angewandte Chemie International Edition 56, 14556-14560, (2017).

34 Oh, Y. et al. An orange calcium-modulated bioluminescent indicator for non-invasive activity imaging. Nature Chemical Biology 15, 433-436, (2019).

Farhana, I., Hossain, M. N., Suzuki, K., Matsuda, T. \& Nagai, T. Genetically Encoded Fluorescence/Bioluminescence Bimodal Indicators for Ca2+ Imaging. ACS Sensors 4, 1825-1834, (2019).

36 Qian, Y., Rancic, V., Wu, J., Ballanyi, K. \& Campbell, R. E. A Bioluminescent Ca2+ Indicator Based on a Topological Variant of GCaMP6s. ChemBioChem 20, 516-520, (2019).

37 Hossain, M. N., Suzuki, K., Iwano, M., Matsuda, T. \& Nagai, T. Bioluminescent Low-Affinity Ca2+ Indicator for ER with Multicolor Calcium Imaging in Single Living Cells. ACS Chemical Biology 13, 1862-1871, (2018).

Yang, J. et al. Coupling optogenetic stimulation with NanoLuc-based luminescence (BRET) Ca++ sensing. Nature Communications 7, 13268, (2016).

Zhang, J.-H., Chung, T. D. Y. \& Oldenburg, K. R. A Simple Statistical Parameter for Use in Evaluation and Validation of High Throughput Screening Assays. Journal of Biomolecular Screening 4, 67-73, (1999).

Schindelin, J. et al. Fiji: an open-source platform for biological-image analysis. Nature Methods $\mathbf{9}$, 676-682, (2012).

41 Lukinavicius, G. et al. Fluorogenic probes for live-cell imaging of the cytoskeleton. Nat Meth 11, 731-733, (2014). 